Bond University

Research Repository

\title{
Delayed prescribing of antibiotics for acute respiratory infections by GP registrars: a qualitative study
}

Dallas, Anthea; Davey, Andrew; Mulquiney, Katie; Davis, Joshua; Glasziou, Paul; Van Driel, Mieke; Magin, Parker

Published in:

Family Practice

DOI:

10.1093/fampra/cmz079

Licence:

Other

Link to output in Bond University research repository.

Recommended citation(APA):

Dallas, A., Davey, A., Mulquiney, K., Davis, J., Glasziou, P., Van Driel, M., \& Magin, P. (2020). Delayed prescribing of antibiotics for acute respiratory infections by GP registrars: a qualitative study. Family Practice, 37(3), 406-411. https://doi.org/10.1093/fampra/cmz079

\section{General rights}

Copyright and moral rights for the publications made accessible in the public portal are retained by the authors and/or other copyright owners and it is a condition of accessing publications that users recognise and abide by the legal requirements associated with these rights.

For more information, or if you believe that this document breaches copyright, please contact the Bond University research repository coordinator 
Title: Delayed prescribing of antibiotics for acute respiratory infections by GP registrars: a qualitative study.

Running head : Delayed antibiotic prescribing by GP registrars

\section{Qualitative research}

\section{Authors}

Anthea Dallas ${ }^{a}$

Andrew Davey ${ }^{b, c}$

Katie Mulquiney ${ }^{b, c}$

Joshua Davis ${ }^{d}$

Paul Glasziou ${ }^{\mathrm{e}}$

Mieke van Driel ${ }^{f}$

Parker Magin ${ }^{\mathrm{b}, \mathrm{c}}$

\section{Affiliations}

a) School of Medicine, University of Tasmania, Hobart, Australia

b) NSW \& ACT Research and Evaluation Unit, GP Synergy, Newcastle, Australia

c) Discipline of General Practice, University of Newcastle, Newcastle, Australia

d) Menzies School of Health Research, Casuarina, Australia

e) Bond University, Gold Coast, Australia

f) Primary Care Clinical Unit, Faculty of Medicine, University of Queensland, Brisbane, Australia

\section{Correspondence:}

Dr A. Dallas, School of Medicine, University of Tasmania, 12 Liverpool St, Hobart, 7000,

Australia; anthea.dallas@utas.edu.au 


\section{Key Messages}

- Delayed prescribing of antibiotics for ARTIs can reduce antibiotic use

- Registrars use it for diagnostic uncertainty, to build rapport or educate patients

- Supervisors recognise the skilled communication needed for these consultations

- Educational intervention should target evidence,appropriate use and communication 


\begin{abstract}
Background:Antibiotic prescribing for acute self-limiting respiratory tract infections (ARTIs) in Australia is higher than international benchmarks. Antibiotics have little or no efficacy in these conditions, and unnecessary use contributes to antibiotic resistance. Delayed prescribing has been shown to reduce antibiotic use. GP registrars are at a career-stage when long-term prescribing patterns are being established.
\end{abstract}

Aim: To explore experiences, perceptions and attitudes of GP registrars and supervisors to delayed antibiotic prescribing for ARTIs.

Design and Setting: A qualitative study of Australian GP registrars and supervisors, using a thematic analysis approach.

Method: GP registrars and supervisors were recruited across three Australian states/territories, using maximum variation sampling. Telephone interviews explored participants' experience and perceptions of delayed prescribing of antibiotics in ARTIs. Data collection and analysis were concurrent and iterative.

Results: Twelve registrars and ten supervisors were interviewed. Key themes included the use of delayed prescribing as a safety-net in cases of diagnostic uncertainty or when clinical review was logistically difficult. Delayed prescribing was viewed as a method of educating and empowering patients, and building trust and the doctor-patient relationship.

Conversely, it was also seen as a loss of control over management decisions. Supervisors, more so than registrars, appreciated the psychosocial complexity of ARTI consultations and the importance of delayed antibiotic prescribing in this context. 
Conclusion: Better awareness and understanding by GP registrars of the evidence for delayed antibiotic prescription may be a means of reducing antibiotic prescribing.

Understanding both registrar and supervisor usage, uncertainties and attitudes should inform educational approaches on this topic.

\section{MeSH terms}

Antimicrobial stewardship

General practice

Graduate medical education

Physician Prescribing Patterns

Qualitative research

Upper respiratory tract infections 


\section{Background}

Antibiotic over-use and subsequent antibiotic resistance have been identified as one of the most important challenges to global health(1). In Australia, approximately $80 \%$ of antibiotic prescription occurs in general practice (2). The most common indication for antibiotic prescription is acute self-limiting respiratory tract infections (ARTIs - such as the common cold, rhinosinusitis, middle ear infections and acute bronchitis) $(3,4)$.

Prescription of antibiotics for these conditions in Australia is inappropriately high $(3,5)$, both by GP registrars (GP vocational trainees) and their more established colleagues $(6,7)$. Antibiotics have relatively little or no efficacy in these ARTIs (8-11), and unnecessary use of antibiotics contributes to increased antibiotic resistance (12). Reducing inappropriate antibiotic use can not only attenuate growth in antibiotic resistance but can also reduce already existing rates of resistance (13).

Delayed antibiotic prescription strategies aim to reduce consumption of antibiotics for acute RTIs in the community. "Delayed prescribing" is when the doctor provides a prescription with advice about if and when to use the prescription (rather than advising immediate antibiotic use). This technique has been shown to reduce antibiotic use for acute ARTIs (compared to "immediate" prescribing), and with similar patient satisfaction (14). While not universally accepted (as an alternative to a policy of 'no prescribing') (15-17) the use of delayed prescribing has been incorporated in international guidelines (18) and is being strongly advocated in Australia $(19,20)$.

GP registrars are an important target for strategies to reduce antibiotic prescription. They are at a stage of their clinical careers when long-term prescribing patterns are being 
developed - there is some evidence that GPs' antibiotic prescribing practices are stable over time (21). Australian GP registrars used "no prescribing" in 68\%, "delayed prescribing" in $9 \%$ and "immediate prescribing" in $23 \%$ of encounters for acute ARTIs (22). This is comparable or higher than the use of delayed prescribing by established GPs in overseas studies $(23,24)$.

Understanding the knowledge and attitudes of GP registrars and their supervisors towards delayed prescribing is important because of the opportunity for education in this phase of training. GP registrar education takes place within an 'apprenticeship-like' model involving a relationship with an experienced GP supervisor (25). The relationship of registrar and supervisor is central to registrars' antibiotic prescribing $(26,27)$, and including the registrarsupervisor dyad in an educational intervention has been shown to reduce inappropriate antibiotic prescribing (28).

This qualitative study aimed to explore GP registrars' and supervisors' knowledge and experiences of, perceptions of, and attitudes to, delayed antibiotic prescribing for selflimiting ARTIs.

\section{Methods}

This was a qualitative study of Australian GP registrars and supervisors, using a thematic analysis approach (29).

\section{Setting}


Australian GP training occurs in (usually) privately-owned practices, where an experienced GP supervises the in-practice training of the registrar. Registrars see patients independently and are responsible for their clinical decision-making but may request assistance and direction from their supervisor as required.

Participating GP registrars and supervisors were recruited from two Regional Training Organizations (RTOs) covering the states of New South Wales, Tasmania and the Australian Capital Territory, and training 35\% of all Australian registrars (30). These RTOs encompass a diversity of urban, regional, rural and remote settings.

\section{Recruitment}

All registrars training with, and supervisors accredited with, the two RTOs were invited by email to participate. Respondents interested in participating completed a demographic questionnaire which facilitated maximum variation sampling (on the bases of gender/Australian or international training/ rurality-urbanicity of practice; and, for registrars, training term; for supervisors, number of years of supervision).

Data collection

Participants were interviewed by telephone by the primary investigator (ADal), using an interview schedule derived from the literature and the study's research questions. The interviews, however, were as much as possible informant-led, allowing new ideas and themes to emerge. Data collection and analysis were concurrent and iterative, with interviews conducted and transcripts analysed during April - September 2018. Questions in 
later interviews were adapted to explore additional findings, and the interview process continued until thematic saturation was achieved (no new themes, relevant ideas or concepts emerging from interviews). Thematic saturation was assessed separately for both registrar and supervisor interviews.

\section{Data analysis}

Interviews were audio-recorded and transcribed verbatim. Data collection and analysis were concurrent and iterative, employing inductive thematic analysis of transcripts and a process of constant comparison to determine emerging themes. Initially, three researchers (ADal, PM, ADav) conducted independent coding, with subsequent comparative coding and the generation of an initial codebook. This codebook was applied to subsequent transcripts and iteratively modified as part of the concurrent data collection and analysis process. At every stage, meanings of participants' responses were discussed, and differences in interpretation between the coding researchers were resolved by discussion.

Codes and themes were collated and then abstracted to form an overarching description of concepts, which was tested against later transcripts as data collection proceeded. The wider research team's expertise in clinical, educational, and content areas (antibiotic prescribing in respiratory tract infections/delayed prescribing) was used to contextualize findings and contribute to the overall interpretation of the data.

Reflexivity was employed at each stage of analysis with an explicit reflection on the professional contexts of the three coding researchers (all GPs, two having in the past few years been GP registrars) and how these may have influenced interpretation of the data. 


\section{Results}

Interviews were conducted in 2018. Thematic saturation was achieved after interviews with a total of twelve GP registrars and ten GP supervisors. See Table 1 for demographic details.

GP registrars' and supervisors' knowledge and experiences of delayed prescribing are outlined, followed by common reasons for choosing a delayed prescribing strategy. Perceptions of positive or negative consequences of delayed prescribing, and attitudes about its use are then described, followed by differences in the supervisor and registrar perspectives on learning about delayed prescribing.

\section{Knowledge and Experience of Delayed Prescribing}

Participant conceptualizations of delayed prescribing entailed both provision of a prescription for antibiotics with instructions to the patient not to use the prescription immediately, and education around if, and when, to fill the prescription and take antibiotics. delayed prescribing is when you sort of prescribe the antibiotic on the day that you see the patient but you tell the patient not to take the antibiotic until the symptoms get worse, and if symptoms are related to a possible bacterial infection. (R1)

Participants frequently described learning of the strategy during formal teaching, at university or as GP registrars, or reading about it. Some, without formal teaching, had noticed other doctors using the strategy.

But I did see a GP do it. I thought that was a good idea. I thought they were a good doctor...they seem like a really conscientious doctor and they're doing this. (R4)

Use of delayed prescribing varied amongst participants. Not all participants endorsed its use, and there was a range of opinions across both registrars and supervisors. Some 
registrars were aware of evidence for delayed prescribing, some weren't. Of those who were aware of evidence supporting use of delayed prescribing, not all translated that knowledge into usual practice.

I don't really do delayed prescribing and I'm not a huge fan of it... I don't think there's much of an evidence base for it (R8)

I know it's a very effective way to reduce the amount of antibiotics people take...I think I've only done it maybe twice...I probably don't do it enough (R4)

When and why to use a delayed prescription

Patient factors such as age (especially young children and the elderly), comorbidities and frailty, severity of symptoms, Aboriginal and Torres Strait Islander status, and rural or remote location or other barriers to healthcare access, were also cited as reasons to consider prescribing (either immediate or delayed).

Another patient factor was the respondents' assessment of patient health literacy.

I am sometimes trying to assess how much medical knowledge they have. On the whole, I think most patients are very good at working out if they're getting worse or not. Some patients I think aren't that good at it and so that will factor into my judgement (S3)

The most common use of delayed prescribing of antibiotics for RTIs was as a "safety net" in the context of diagnostic uncertainty. Participants usually expressed a preference to review 
the patient in the case of deterioration, but, if that were not possible, offering a delayed prescription was an alternative to this. Many participants described logistical or geographical reasons for the use of delayed prescribing:

... it's a weekend, the long weekend, they're about to go away or they're travelling on a cruise or something, so they might be reasons why I would give them a script to use if needed. (S3)

In describing the clinical reasoning around the decision to provide a delayed prescription, GPs often spoke of a dichotomous decision about whether the illness was of viral or bacterial aetiology, plus concerns of progress from a viral infection to a secondary bacterial infection:

But for ones we think there's a chance that it will progress or that it's sort of on the edge and you're not quite sure if it is viral or if it is already bacterial-sometimes we do give them antibiotics then get them to hang on and see how the next two days go and if you're getting worse take the antibiotics. If you're getting better, don't take the antibiotics (S8)

\section{Diagnostic uncertainty as a driver of delayed prescribing}

Diagnostic uncertainty (including uncertainty as to bacterial/viral aetiology) was a major reason given by participants to consider delayed prescribing. This uncertainty was sometimes related to the stage of the presentation, concerns that it might progress, clinician inexperience, and previous negative experiences (such as a missed diagnosis). 
With bronchitis or early pneumonia or something, it's hard to tease out... yeah, delayed prescribing is helpful. (R7)

But I feel like I'm probably more likely to give antibiotics after I feel like I have missed giving antibiotics, for want of a better way to put it, and in children, because I am less confident in kids than I am in adults. (R4)

The GP's affective response to uncertainty, and their tolerance for the anxiety that uncertainty provoked, was an important theme. Most participants cited specific instances where "something had gone wrong" as influencing their subsequent prescribing practice:

They've got a full-blown pneumonia and they're just really sick and I feel terrible and I feel that this really shouldn't have happened. Those are the people that I'm really trying to avoid this happening to with the delayed antibiotic prescribing. (S3)

Clinical experience, however led to changes in participants' tolerance for uncertainty and, thence, to changes in practice:

When you've seen so many clinical presentations... know you can never be $100 \%$ sure, but you can be a bit more confident about it than probably I was, say, 20 years ago. (S9) ...as I become a bit more confident in my diagnosing, I probably do give a bit less. (R8)

Perceptions and attitudes: is delayed prescribing a positive or negative strategy? 
Delayed prescribing sometimes was perceived positively - as an opportunity to educate and empower patients:

You want [patients] to be happy, but certainly over the long term you want them to become more health literate and provide self-care for themselves. (S7)

...you're allowing the patient to have a bit of judgement in the whole process, particularly a parent. (S9)

Developing and maintaining the doctor-patient relationship was also perceived as a positive outcome of delayed prescribing. A registrar suggested delayed prescribing with education is a better option than a disgruntled patient seeing another GP:

when I say "I'm not giving you the script," a lot of the times what happens is they come back to another GP and get the script and there's no - there's no sort of education or they're not thinking whether they need it or not. (R12)

Offering a delayed prescription could be a method of compromise to meet patient expectations in certain situations. This compromise was mostly not seen as a positive aspect of delayed prescribing. Such action was generally viewed as an unsatisfactory compromise, with GPs describing a feeling of "giving in" or "failure" when using this strategy.

Well, I would say that it's probably often used by people as a bit of a get-out-of-jailfree card .... feel like that's sort of failing a little bit in that if they're not meant to have antibiotics, you haven't given really a very clear answer there and a clear message to the patient.(R5) 
Other negative consequences of delayed prescribing included the sense of relinquishing control of the treatment decision (as opposed to the 'positive' patient empowerment felt by some participants), and the potential for inappropriate future use of unfilled prescriptions.

[It's] relinquishing control and hoping that parents make the right decision. The thing I fear is the situation worsening and them starting antibiotics when the child should really be reassessed instead ( $R 11)$

They didn't get it filled initially and then they filled it later, or someone in the family filled it, that's a problem. You've got this live prescription floating around. (S9)

\section{Trust and Relationship}

Overarching these themes of education, empowerment and compromise, was the broader concept of trust between GP and patient:

If you've got a sensible person who's fully cognisant of the fact that they need to wait and most likely it's going to settle down, and you trust them that they will do the right thing, I'd probably still prescribe and have delayed prescribing that way. (S9) It kind of depends on how I feel the parents respond to my advice, and if I know that they're taking it on board correctly, and we've got, I suppose, a good rapport (R10) This theme of the "sensible" or "trustworthy" patient was more prevalent amongst supervisors, who had long-term relationships with their patients.

\section{Learning about the psychosocial complexity of delayed prescribing}


Registrars frequently reported not asking supervisors for advice around prescribing decisions for ARTIs, feeling that they were "too simple" and not requiring supervisor input. I would go in to talk to my supervisor about the difficult diabetes or whatever rather than the five viral URTIs ...it seems more common, so I don't need to ask that. (R7)

But when supervisors were asked what they wished to impart to registrars on the topic of delayed prescribing, they focused on the psychosocial (rather than biological) complexity of decision-making around delayed antibiotic prescribing, including communication and consultation skills, and how to apply evidence in the real-life context of a patient.

Rather than...this is what the book says you do - what were they going to do in this sort of setting and this context? (S9)

I think for registrars it's mostly about confidence and language that they use to be able to actually [engage a person about treatment]. I don't think it's impossible to learn them, because they are real skills...It's about teaching those skills. (S6)

\section{Discussion}

\section{Summary of findings}

Key themes in our findings included the use of delayed prescribing as a safety-net, especially in cases of diagnostic uncertainty or when review was logistically difficult. The GP's affective response to uncertainty was important. Most participants reported that, with experience and consequent clinical confidence, feelings of uncertainty and anxiety lessened 
and there was less need for delayed prescribing as response to uncertainty. Delayed prescribing was viewed as a method of educating and empowering patients, building trust and relationship with the doctor or, conversely, as a loss of control over management decisions. We found that supervisors, in contrast to registrars, better recognised the complexity of making a delayed prescribing decision, in particular the psychosocial factors of the consultation. This led supervisors to spend time seeking to impart communication skills to their registrars.

\section{Strengths and Limitations}

The use of qualitative methods allowed us to focus on the contextual factors surrounding a decision to use delayed prescribing, which has been noted to be an important requirement in understanding the complexity of antibiotic prescribing (31). Furthermore, we approached this question from the perspective of both GP registrars and supervisors, giving further depth to the understanding of the experience of the GP-in-training (during an important period for establishing clinical practice patterns).

Our sample frame comprised a broad range of Australian GP registrars and their supervisors. The transferability to early-career doctors in other countries will be affected by contexts of the particular health systems and, especially, by the similarity of training systems to the Australian apprenticeship-like model.

\section{Implications of findings for clinical and educational practice}

Antibiotic prescribing can be understood as a medical, social and cultural practice (31), and our study describes the particular context of the GP registrar as they move through training. Antibiotic prescribing is culturally influenced (13) and considering the Australian context 
adds to the international understanding of this global problem (15). Australian guidelines at the time of the study (32) did not specifically mention delayed prescribing, unlike (for example) the NICE guidelines in the UK (18). There has been, however, advocacy for the increased use of delayed prescribing in Australian general practice (20), given that there is an imperative for reduction in antibiotic prescribing (3).

The decision to prescribe an antibiotic for an ARTI is rarely made on clinical factors alone $(31,33-35)$, and this also applies to the decision to use a delayed antibiotic prescription (15, 17). The relationship of trust between GP and patient and its effect on the use of delayed prescribing has been noted in other studies $(16,36,37)$. The specific "non-clinical" factors that are applicable to GP registrars are important findings in our study - in particular, lack of experience and heightened sense of diagnostic uncertainty for the early-career $\mathrm{GP}(26,38$, 39), and a lack of longitudinal relationship with patients that is inherent in their situation (36). Participants' perceived need to distinguish viral/bacterial aetiology in self-limiting ARTIs is not evidence-based and may compound diagnostic uncertainty. This is an appropriate topic for registrar education (40).

\section{Conclusion}

Educating GP registrars to feel confident in the use of delayed antibiotic prescribing for ARTIs may assist in combating antibiotic resistance, especially as registrars struggle with diagnostic uncertainty more than their established colleagues. Our findings suggest that this education should focus on the common concerns for registrars, and the psychosocial complexity of these prescribing decisions which require skilled communication. 


\section{Declarations}

Ethics: Ethics approval was granted by the Royal Australian College of General Practitioners

Ethics Committee (Reference: NREEC 17-014)

Funding: Funded by Royal Australian College of General Practitioners Foundation FMCER

Grant 2017 (Reference: FMC17-09)

Conflict of Interest: none to report

Acknowledgements: We are grateful to all participants, and to the GP Synergy and GPTT staff for their assistance.

\section{References}

1. OECD. Stemming the Superbug Tide: Just A Few Dollars More. Paris: OECD Publishing; 2018.

2. Del Mar C, Glasziou P, Lowe JB, van Driel ML, Hoffmann T, Beller E. Addressing antibiotic resistance - focusing on acute respiratory infections in primary care. Australian family physician. 2012;41(11):839-40. 
3. McCullough AR, Pollack AJ, Plejdrup Hansen M, Glasziou PP, Looke DF, Britt HC, et al. Antibiotics for acute respiratory infections in general practice: comparison of prescribing rates with guideline recommendations. The Medical journal of Australia. 2017;207(2):65-9.

4. Shallcross LJ, Davies DS. Antibiotic overuse: a key driver of antimicrobial resistance. British Journal of General Practice. 2014;64(629):604-5.

5. Pan Y, Henderson J, Britt H. Antibiotic prescribing in Australian general practice: how has it changed from 1990-91 to 2002-03? Respir Med. 2006;100(11):2004-11.

6. Dallas A, Magin P, Morgan S, Tapley A, Henderson K, Ball J, et al. Antibiotic prescribing for respiratory infections: a cross-sectional analysis of the ReCEnT study exploring the habits of earlycareer doctors in primary care. Family practice. 2015;32(1):49-55.

7. Magin PJ, Morgan S, Tapley A, Henderson KM, Holliday EG, Ball J, et al. Changes in earlycareer family physicians' antibiotic prescribing for upper respiratory tract infection and acute bronchitis: a multicentre longitudinal study. Family practice. 2016;33(4):360-7.

8. Venekamp RP, Sanders S, Glasziou PP, Del Mar CB, Rovers MM. Antibiotics for acute otitis media in children. Cochrane Database Syst Rev. 2013;1:CD000219.

9. Spinks A, Glasziou P, DelMar C, Issue 11. . Antibiotics for sore throat. Cochrane Database Syst Rev. 2013; CD000023.

10. Smith SM, Fahey T, Smucny J, Becker LA. Antibiotics for acute bronchitis. [Update of Cochrane Database Syst Rev. 2004;(4):CD000245; PMID: 15494994]. Cochrane Database Syst Rev. 2014;3:CD000245.

11. Kenealy T, Arroll B. Antibiotics for the common cold and acute purulent rhinitis. Cochrane Database Syst Rev. 2013;6:CD000247.

12. Costelloe C, Metcalfe C, Lovering A, Mant D, Hay AD. Effect of antibiotic prescribing in primary care on antimicrobial resistance in individual patients: systematic review and meta-analysis. Bmj. 2010;340:c2096.

13. Goossens $\mathrm{H}$, Ferech $\mathrm{M}$, Van der Stichele R, al e. Outpatient antibiotic use in Europe and association with resistance: a cross-national database study. . Lancet. 2005;365(9459):579-87. 14. Spurling GK, Del Mar C, Dooley L, Foxlee R, Farley R. Delayed antibiotic prescriptions for respiratory infections. (Review). Cochrane Database Syst Rev. 2017;CD004417.

15. Peters S, Rowbotham S, Chisholm A, Wearden A, Moschogianis S, Cordingley L, et al. Managing self-limiting respiratory tract infections: a qualitative study of the usefulness of the delayed prescribing strategy. Br J Gen Pract. 2011;61(590).

16. Ryves R, Eyles C, Moore M, McDermott L, Little P, Leydon GM. Understanding the delayed prescribing of antibiotics for respiratory tract infection in primary care: a qualitative analysis. BMJ open. 2016;6(11):e011882.

17. Sargent L, McCullough A, Del Mar C, Lowe J. Using theory to explore facilitators and barriers to delayed prescribing in Australia: a qualitative study using the Theoretical Domains Framework and the Behaviour Change Wheel. BMC family practice. 2017;18(1):20.

18. Centre for Clinical Excellence, Respiratory tract infections - antibiotic prescribing. London: National Institute for Health and Clinical Excellence; 2008. Available from: https://www.nice.org.uk/guidance/cg69/evidence/full-guideline-pdf-196853293.

19. McCullough A, Glasziou P. Delayed Antibiotic Prescribing Strategies-Time to Implement? JAMA internal medicine. 2016;176(1).

20. Sargent L, McCullough A, Del Mar C, Lowe J. Is Australia ready to implement delayed prescribing in primary care? A review of the evidence. Australian family physician. 2016;45(9):68890.

21. Bjornsdottir I, Kristinsson KG, Hansen EH. Diagnosing infections: a qualitative view on prescription decisions in general practice over time. Pharm World Sci. 2010;32(6):805-14.

22. Davey A, Tapley A, van Driel M, Glasziou P, Dallas A, Davis J, et al. Antibiotic prescribing strategies of general practice registrars for acute respiratory tract infections: the role of delayed 
prescribing. . General Practice Training and Education Conference Sydney August 2017. Available at: https://research.gpsynergy.com.au/publications/

23. Francis N, Gillespie D, Nuttall J, Hood K, Little P, Verheij T, et al. Delayed antibiotic prescribing and associated antibiotic consumption in adults with acute cough. Br J Gen Pract. 2012;62(602).

24. Godycki-Cwirko M, Hood K, Nocun M, Muras M, Goossens H, Butler CC. Presentation, antibiotic management and associated outcome in Polish adults presenting with acute cough/LRTI. . Family practice. 2011;28(608-614).

25. Thomson JS, Anderson KJ, Mara PR, Stevenson AD. Supervision--growing and building a sustainable general practice supervisor system. The Medical journal of Australia. 2011;194(11):S1014.

26. Dallas $A$, van Driel $M$, van de Mortel $T$, Magin $P$. Antibiotic prescribing for the future: exploring the attitudes of trainees in general practice. Br J Gen Pract. 2014;64(626):e561-7.

27. Papoutsi C, Mattick K, Pearson M, Brennan N, Briscoe S, Wong G. Interventions to improve antimicrobial prescribing of doctors in training (IMPACT): a realist review. Health Services and Delivery Research. Southampton (UK): NIHR Journals Library

28. Magin P, Tapley A, Morgan S, Davis JS, McElduff P, Yardley L, et al. Reducing early career general practitioners' antibiotic prescribing for respiratory tract infections: a pragmatic prospective non-randomised controlled trial. Family practice. 2018;35(1):53-60.

29. Braun V, Clarke V. Using thematic analysis in psychology. Qualitative Research in Psychology. 2006;3:77-101.

30. Taylor R, Radloff A, Edwards D, Clarke L. National report on the 2017 National Registrar Survey. (2018) Available at: http://www.agpt.com.au/ArticleDocuments/324/Australian-GeneralPractice-Training-Program-\%20National-report-on-the-2016-Registrar-Satisfaction-Survey.pdf.aspx 31. Ackerman S, Gonzales R. The context of antibiotic overuse. Annals of internal medicine. 2012;157(3):211-2.

32. Therapeutic Guidelines: Antibiotic. Melbourne: Therapeutic Guidelines Limited; 2014.

33. Petursson P. GPs' reasons for "non-pharmacological" prescribing of antibiotics. A phenomenological study. Scandinavian journal of primary health care. 2005;23(2):120-5.

34. Lucas PJ, Cabral C, Hay AD, Horwood J. A systematic review of parent and clinician views and perceptions that influence prescribing decisions in relation to acute childhood infections in primary care. Scandinavian journal of primary health care. 2015;33(1):11-20.

35. Strumilo J, Chlabicz S, Pytel-Krolczuk B, Marcinowicz L, Rogowska-Szadkowska D, Milewska AJ. Combined assessment of clinical and patient factors on doctors' decisions to prescribe antibiotics. BMC family practice. 2016;17:63.

36. Strandberg EL, Brorsson A, Hagstam C, Troein M, Hedin K. "I'm Dr Jekyll and Mr Hyde": are GPs' antibiotic prescribing patterns contextually dependent? A qualitative focus group study. Scandinavian journal of primary health care. 2013;31(3):158-65.

37. Germeni E, Frost J, Garside R, Rogers M, Valderas JM, Britten N. Antibiotic prescribing for acute respiratory tract infections in primary care: an updated and expanded meta-ethnography. BJGP. 2018;68(674):e633-e45.

38. Horwood J, Cabral C, Hay AD, Ingram J. Primary care clinician antibiotic prescribing decisions in consultations for children with RTIs: a qualitative interview study. BJGP. 2016;66(644):e207-13.

39. Nevalainen M, Kuikka L, Pitkala K. Medical errors and uncertainty in primary healthcare: a comparative study of coping strategies among young and experienced GPs. Scandinavian journal of primary health care. 2014;32(2):84-9.

40. Davis J, Magin P, van Driel $M$. The false dichotomy of viral versus bacterial aetiology in upper respiratory tract infections. Medical Journal of Australia 2019. 
Table 1: Demographics of participants

GP registrars/supervisors from participating Regional Training Organisations

Data collected 2018

\begin{tabular}{llllll}
\hline $\begin{array}{l}\text { Participant } \\
\text { code }\end{array}$ & $\begin{array}{l}\text { Supervisor/ } \\
\text { Registrar }\end{array}$ & Gender & $\begin{array}{l}\text { Years as } \\
\text { supervisor/ } \\
\text { GP training } \\
\text { term (GPT) }\end{array}$ & $\begin{array}{l}\text { Urban/Rural classification } \\
\text { of practice location }\end{array}$ & $\begin{array}{l}\text { International } \\
\text { (IMG) or } \\
\text { Australian } \\
\text { (Aus) graduate }\end{array}$ \\
\hline S1 & Supervisor & F & 1 & Major City & IMG \\
\hline S2 & Supervisor & M & 1 & Outer Regional & Aus \\
S3 & Supervisor & F & 2 & Major City & Aus \\
S4 & Supervisor & M & 2 & Major City & Aus \\
S5 & Supervisor & M & 2 & Outer Regional & Aus \\
S6 & Supervisor & F & 3 & Major City & Aus \\
S7 & Supervisor & M & 4 & Major City & Aus \\
\hline S8 & Supervisor & F & 14 & Outer Regional & Aus \\
S9 & Supervisor & M & 20 & Inner Regional & Aus \\
\hline S10 & Supervisor & F & 20 & Major City & Aus \\
\hline R1 & Registrar & M & GPT1 & Inner Regional & IMG \\
\hline R2 & Registrar & F & GPT1 & Inner Regional & Aus \\
R3 & Registrar & M & GPT1 & Major City & Aus \\
\hline R4 & Registrar & F & GPT2 & Major City & Aus \\
\hline R5 & Registrar & F & GPT2 & Inner Regional & IMG \\
R6 & Registrar & M & GPT3 & Major City & Aus \\
\hline R7 & Registrar & F & GPT3 & Major City & Aus \\
\hline R8 & Registrar & M & GPT3 & Major City & Aus \\
R9 & Registrar & F & GPT3 & Outer Regional & Aus \\
\hline R10 & Registrar & F & GPT4 & Inner Regional & IMG \\
R11 & Registrar & F & GPT4 & Major City & Aus \\
\hline R12 & Registrar & F & GPT4 & Major City & Aus \\
\hline & & & & & \\
\hline
\end{tabular}

